\title{
Kunstunterrichtliches Lernen im Spannungsfeld von Kind und Kunst - Zur inklusiven Bildung und kunstunterrichtlichem Lernen in der Grundschule
}

\author{
Michaela Kaiser (D) Andreas Brenne
}

Eingegangen: 8. Juni 2020 / Angenommen: 11. Oktober 2020 / Online publiziert: 22. Februar 2021

(C) Der/die Autor(en) 2021

Zusammenfassung Dieser Beitrag bearbeitet überblicksartig inklusives Lernen in der Grundschule aus kunstdidaktischer Perspektive. Ausgehend von der Auffassung, dass der Kunstunterricht und dessen künstlerische Bezüge für eine inklusive Perspektive nahezu prädestiniert zu sein scheinen, paradoxerweise aber die Diskussion um inklusive Bildung in kaum einer Fachdidaktik so wenig systematisch aufgegriffen wurde, werden kunstdidaktische Diskurse strukturell beleuchtet, um einen möglichen Perspektivwechsel vorzubereiten. Unter dieser Prämisse wird der Frage nachgegangen, welche Bezüge zum inklusiven fachlichen Lernen sich im Kunstunterricht nachzeichnen lassen und inwiefern dieser Zusammenhang im Kunstunterricht der Grundschule thematisiert wird.

Schlüsselwörter Kunstunterricht · Inklusion · Grundschule

Dr. M. Kaiser $(\bowtie)$

Universität Paderborn, Warburger Straße 100, 33098 Paderborn, Deutschland

E-Mail: michaela.kaiser@uni-paderborn.de

Prof. Dr. A. Brenne

Universität Potsdam, Karl-Liebknecht-Straße 24-25, 14476 Potsdam, Deutschland 


\title{
Inclusive art education in primary schools
}

\begin{abstract}
This article deals with inclusive learning in primary schools from an art educational perspective. Art education seems predestined for the development of an inclusive perspective, but at the same time the discussion about inclusive education has paradoxically not been taken up systematically. Therefore, art didactic discourses are structurally shed light on in order to provide a possible change of perspective. Based on this assumption, we will investigate which aspects of inclusive learning can be traced in art education and to what extent this is addressed in primary school art classes.
\end{abstract}

Keywords Art education · Inclusion · Primary school

\section{Einleitung}

In kaum einer Fachdidaktik wurde die Diskussion um eine inklusive Bildung so spät aufgegriffen wie in der Kunstdidaktik, gleichwohl der Kunstunterricht und dessen künstlerische Bezüge für eine inklusive Perspektive prädestiniert zu sein scheinen (Brenne 2016). Nicht nur, weil in den künstlerischen Wahrnehmungs- und Handlungsvollzügen differente Perspektiven eine substantielle Bedeutung haben und darüber hinaus für künstlerische Rezeptions- und Produktionsprozesse unerlässlich sind (ebd.), sondern vor allem, da der Kunstunterricht aufgrund dessen nur als zieldifferenter Unterricht denkbar sei (Griebel 2017), hat sich die landläufige Meinung etabliert, dass Inklusion und Kunstunterricht zwei Seiten desselben Phänomens darstellen. So verwundert es umso mehr, dass diese Auffassung bisher nur marginal in der kunstunterrichtlichen Forschung Beachtung findet. Im Kontext der Kunstpädagogik der Grundschule, in der immer noch eine solipsistische Vermittlung deklarativen Wissens dominiert, ist dies umso bemerkenswerter, da es kaum dem pädagogischen Profil der Grundschule zu entsprechen scheint, die zum einen die pädagogische Dimension für zentral erachtet und zum anderen das gemeinsame Lernen heterogener Gruppen als konstitutiv versteht. Die Arbeit mit heterogenen Lerngruppen ist hier phänomenal geboten: Kindheiten werden nicht nur als unterschiedlich gefasst, sondern auch als ungleich, wovon Kinder in unterschiedlicher Weise betroffen sind (Huf und Schnell 2015). Unter Rekurs auf Prengel resümiert Kucharz (2015) von daher, dass die „Diskussion über eine Pädagogik der Vielfalt, die Verschiedenheit aufnehmen und nutzen will, ohne einzelne Kindergruppen zu marginalisieren oder zur Assimilation zu zwingen, [...] in der Grundschulpädagogik schon seit 20 Jahren geführt" wird. Hier wird demnach schon seit längerem eine neue Lernkultur verortet, in der eine ,kindfähige' Schule individuelle Lernwege und innere Differenzierung in den Fokus unterrichtlicher Bemühungen stellt.

Vergleicht man die hier angesiedelte Kunstdidaktik mit anderen Fachdidaktiken, ist eine vergleichsweise zähe Transformation allgemeindidaktischer Wissensbestände $\mathrm{zu}$ verzeichnen. Zwar liegt eine vielfältige Kompendienliteratur vor (Ehmer 2003), die sich aber zumeist in einer aufgabenorientierten Vermittlung bild- respektive kunstorientierter Kompetenzen erschöpft und Aspekte der Differenzierung 
wie auch einer kollaborativen Justierung fachlicher Inhalte ausspart. Auch die programmatischen Schriften des Fachverbandes für Kunstpädagogik [BDK] beziehen zum inklusiven Lernen bisher kaum Stellung (BDK 2001). Insofern scheint die Vermittlung deklarativen Wissens im Kontext der Grundschule zentral zu sein, obwohl es durchaus Tendenzen gab das ästhetische Lernen als subjektorientiertes Lernprogramm im Kontext der Grundschule zu etablieren (Staudte 1993). Die strukturelle Konzentration auf den innerfachlichen Diskurs wandelte sich im Kontext erster Annäherungen an den Inklusionsdiskurs.

So wurden didaktische Fragen zunächst eng auf die erziehungswissenschaftliche Inklusionsforschung bezogen (Loffredo 2016), während darauffolgende deutschsprachige Publikationen (Blohm et al. 2017; Engels 2017a; Hornäk et al. 2019; Kaiser 2019; Penzel 2017) zusehend mehr Impulse aus der Kunstdidaktik selbst ableiteten. Die Auseinandersetzungen weisen darauf hin, dass die Bestimmung einer inklusiven Kunstdidaktik im Hinblick auf die zentralen Aufgabenbereiche des Faches ein dialektisches Spannungsverhältnis zwischen erziehungswissenschaftlichen und fachlichen Bezügen sichtbar macht. $\mathrm{Zu}$ nennen sind

- die Entwicklung bildanalytischer Kompetenzen im Sinne der Wahrnehmung und kritischen Analyse künstlerisch-gestalterischer Ausdrucksformen als Voraussetzung einer selbstbestimmten und aktiven Teilhabe an Kunst und Kultur und

- die Entwicklung gestalterischer Kompetenzen, die darauf abzielt mittels eigener gestalterischer Praxis kreatives Potenzial zu erkennen und zu entfalten und somit eine eigene künstlerische Identität zu entwickeln, wodurch Persönlichkeitsbildung unterstützt wird.

Zum einen wird die kunstdidaktische Debatte entsprechend ihrer fachlichen und erziehungswissenschaftlichen Bezüge im Hinblick auf Ziele und Inhalte des Fachs Kunst und zum anderen im Hinblick auf die methodische Umsetzung des Unterrichts geführt. Aus diesem Blickwinkel führte die didaktische Diskussion zu einer Fokussierung von Prinzipien inklusiver (Seitz 2006, 2008, 2020; Ziemen 2014) bzw. integrativer Didaktik (Feuser 1989; Wocken 2015), mit denen die grundlegende Frage nach Individualisierung und Differenzierung aufgenommen wird, sich aber vielgestaltig ausdifferenziert. Eine Orientierung an solchen didaktischen Merkmalen kann die Bandbreite der Heterogenität kindlicher Lernausgangslagen einfassen, weshalb sie zugleich auch als ,gute“ allgemeine Didaktik begriffen wird (Lütje-Klose 2018); und umgekehrt wird die anvisierte grundlegende Bildung in der Grundschule gar als Referenz für eine inklusive Bildung herangezogen (Vogt 2019). Doch muss gefragt werden, wie sich diese Prämissen auf die fachliche Ausrichtung des Unterrichts auswirken, genau genommen, wie die Lerninhalte im Fach Kunst zu bestimmen sind.

\section{Zwischen Kind und Kunst: Kunstdidaktische Perspektiven}

Bisher wurde die kunstdidaktische Diskussion aufgrund der spezifischen disziplinären Bezüge des Kunstunterrichts, insbesondere und vor allem zur Kunst, selten in enger Beziehung zur Grundschulpädagogik geführt. Wichtige Impulse kamen - wie 
bereits ausgeführt - dazu aus den Diskursen zur ästhetischen Erziehung (Staudte 1993), aus denen heraus die Orientierung am Individuum und die damit einhergehende grundlegende Subjektorientierung des Kunstunterrichts hervorging, die Basis für eine Neuorientierung der Lernkultur sein sollte. Sie rückt die Fragen der Kinder selbst in den Mittelpunkt des Kunstunterrichts. Neuere Konzeptionen verfeinern, was sich auch in einer kunstpädagogischen Konzeptbildung für die Grundschule niederschlägt. Hervorzuheben sind Formen einer ,forschenden Kunst", die ihren Bezug zum einen in einer derart ausgerichteten Kunst wiederfindet (Brenne 2004) und sich zum anderen am Prinzip des forschenden Lernens orientieren. Dabei werden künstlerische Verfahren, insbesondere im Hinblick auf eine phänomenal an der Kunst orientierten Kunstpädagogik (Kirchner 1999, 2013) zum Gegenstand unterrichtlicher Bemühungen, wodurch die gängige Dichotomie zwischen den Kernbereichen von Rezeption und Produktion funktional verknüpft wird; wobei Kunstwerke im Rahmen einer formanalytischen Reflexion zum Initial einer weiterführenden eigenständigen Gestaltung herangezogen werden, so dass sich Rezeption und Produktion unter der Prämisse ihrer doppelseitigen Erschließung miteinander verzahnen. Die Kunstrezeption selbst liefert Impulse für eine mimetische (ebd.), aber auch für eine philosophisch-erfahrungsgeleitete gestalterische Transformation, die sich stärker an künstlerischen Narrationen orientieren (Uhlig 2005). Mit der Begründung einer partizipatorisch angelegten Kunstpädagogik in der Grundschule (Brenne et al. 2013; Urlaß et al. 2012) wird daneben der Grundstein für einen explizit an Teilhabe orientierten Kunstunterricht gelegt. Insgesamt wurden prozessorientierte und partizipatorische künstlerische Wahrnehmungs- und Handlungsvollzüge für den Kunstunterricht der Grundschule zunehmend ausdifferenziert und finden sich aktuell in Projekten wie die ,Grundschule der Künste“ (Winderlich 2019), in welcher derartige Prinzipien in einem offenen, experimentellen und kollaborativen Setting erprobt, ausgewertet und in konkrete Unterrichtsvorschläge transformiert werden. Inklusive Unterrichtsformen sind hier quasi systemimmanent, ohne konkret benannt zu werden.

Doch findet sich noch vielfach eine Beschränkung des Fachs Kunst auf fachspezifische Themenbereiche, während eine Vernetzung von fachspezifischen und fachübergreifenden Domänen die Ausnahme darstellt - gleichwohl der Kunstunterricht so gedacht als künstlerisch und ästhetisch emanzipierte Erschließung von Lebens- und Umwelt konzeptualisiert werden kann (dazu Brenne 2004; Kämpf-Jansen 2001). Zugleich stellt sich mit einem Blick auf die grundlegenden Inhaltsbereiche des Kunstunterrichts die Frage, wie Lern- und Bildungsprozesse auch in geschlossenen Aufgabenformaten zur Entfaltung kommen können. Bildorientierte (Bering 2006) und gestaltungsdidaktische (Glas und Sowa 2006) Ansätze können hierauf Antworten geben. Die Zielsetzung der bereits implizit in den 1960er-Jahren entwickelten Kernmethode der Bildorientierung (u.a. Otto; Pfennig) ist die Förderung von Bildliteralität und die Ausbildung informierter und mündiger Kunstrezipientinnen und Kunstrezipienten (Duncker und Lieber 2013), wobei Bering (2006) auch den produktiv-gestalterischen Aspekt einbezieht. Hingegen erfahren ästhetisch-geistige Aneignungsformen von Lebenswelt in der Gestaltungsdidaktik eine gehobene Aufmerksamkeit, denn über den Erwerb gestalterischer Expertise hinaus geht es hierbei auch und vor allem um die Erschließung von Weltphänomenen (Glas und 
Sowa 2006, S. 261) - gestalterisches Wissen ist hier als eine individuelle Kulturleistung aufzufassen (Sowa 2017), was angesichts der hohen kulturellen Diversität der Grundschule und vor dem Hintergrund der inklusiven Herausforderungen an den Kunstunterricht spezifische Impulse zu setzen vermag. Die an der Kunst orientierte künstlerische Kunstpädagogik rückt hingegen das Verhältnis zwischen dem Individuum und der Gesellschaft stärker in das Blickfeld, weshalb eine Vermittlung formaler Aspekte von Kunst als unzulänglich erachtet wird (Buschkühle 2009). Ziel ist die Vermittlung künstlerischer Denk- und Handlungsweisen zur Veränderung und Mitgestaltung von Welt und Umwelt. Indem sich eine künstlerische Kunstpädagogik an künstlerischen Methoden und Verfahren ausrichtet, werden thematisch disparate Praxen eingeübt und sukzessive etabliert. Unter Rückbezug auf den Gedanken der sozialen Plastik nach Beuys rückt die künstlerische Kunstpädagogik einen humanistischen Kunst- und Bildungsbegriff in ihr Zentrum, wodurch konsequent an die Motivlagen von Kindern angeknüpft wird (Engels 2017b; Griebel 2017). Hinzu kommen Methoden einer ästhetisch-künstlerischen Forschung, mittels derer lebensweltliche Phänomene interdisziplinär untersucht werden (Brenne 2004; KämpfJansen 2001). Der künstlerischen (Brenne 2004, 2017b) und ästhetischen (KämpfJansen 2001) Forschung ist der Subjektbezug ebenso inhärent wie eine hohe Fachlichkeit, womit diese konsequent einer didaktischen Orientierung am Kind folgen. Die Konzepte sind konstruktivistisch angelegt und beanspruchen die Vielfalt der lebensweltlichen und soziokulturellen Bezüge zum Ausgangspunkt von kunstunterrichtlichen Lernarrangements zu machen. Ziel ist die Entwicklung einer ästhetischforschenden Haltung, aus der heraus die Beziehung zwischen Person und Umwelt auf Basis individueller Lesarten und Bildsprachen untersucht und neu ausgelotet wird (Brenne 2006). Diese Form der forschenden Ausrichtung des Kunstunterrichts hat in einem inklusiven Unterricht einen direkten Bezug zu den drei Prinzipien der Personalität, Sozialität und Komplexität (Seitz 2020) und wurde im Sinne eines Perspektivwechsels zwar breit rezipiert, bislang jedoch noch nicht curricular aufbereitet. Aus der Perspektive der bildorientierten Kunstpädagogik und der Gestaltungsdidaktik wird kritisch ausgeführt, dass derart kunst- und biografieorientierte Ansätze die Frage nach der Entwicklung von Kompetenzmodellen und differenzierten Methoden strukturell verhindern (Loffredo 2016; Penzel 2017). Unabhängig von diesem konzeptionellen und paradigmatischen Widerstreit innerhalb der Kunstpädagogik sollte die Frage nach den spezifischen Qualitäten eines inklusiven Kunstunterrichts anders gestellt werden: So geht es nicht um die Legitimität der eigenen Position im Hinblick auf künstlerische oder kunstwissenschaftliche Wahrheitswerte, sondern um die Frage, wie diese Zielsetzungen sich im Rahmen von Lernarrangements entfalten, die auf einer prinzipiellen und hierarchiefreien Anerkennung heterogener Perspektiven und Befähigungen beruhen.

\section{Fachdidaktische Perspektiven auf inklusiven Kunstunterricht}

Vor dem Hintergrund der vorausgegangenen Überlegungen geht es im Weiteren um die Frage, wie Grundlagen einer inklusiven Kunstdidaktik im Allgemeinen und für die Grundschule im Spezifischen aussehen könnten. 


\subsection{Entwicklungslinien inklusiven fachlichen Lernens im Kunstunterricht}

Eine differenzierende und in gleicher Weise differenzierte Förderlandschaft hat im Kontext einer heilpädagogischen Kunstpädagogik bereits eine lange Tradition. Insbesondere Theunissen (2013) hat die Frage nach der Ressource Kunst in vielfältiger Weise gestellt und konnte zu einer spezifizierten Beantwortung im Hinblick auf unterschiedliche Behinderungsarten und Förderschwerpunkte kommen, die auch in die schulischen Konzeptualisierungen einer sonderpädagogischen Kunstpädagogik Eingang gefunden haben. Trotz des hinterlegten Verständnisses nehmen einige seiner fachlichen Ansätze bis heute Einfluss auf den kunstpädagogischen Inklusionsbegriff und kunstdidaktische Planungs- und Handlungsmodelle, die einem weiten Inklusionsbegriff folgen, sind erst seit kurzem Gegenstand der kunstpädagogischen Diskussion. Damit lässt sich konstatieren, dass trotz der inklusiven Bildungsvalenzen kunstunterrichtlicher Ansätze die Inklusionsdebatte im Hinblick auf kunstunterrichtliches Lernen im Vergleich mit anderen Fachdidaktiken nicht nur spät aufgegriffen wurde, sondern auch auf wenige aktive Autorinnen und Autoren beschränkt geblieben ist (Brenne 2016; Blohm et al. 2017; Hornäk et al. 2019; Engels 2017a; Kaiser 2019; Loffredo 2016).

$\mathrm{Zu}$ einer fachdidaktischen Bearbeitung der Inklusionsthematik im Kontext der deutschsprachigen Kunstpädagogik forderte zunächst Engels (2015) in den programmatischen Mitteilungen des BDK auf, wenn sie aus einer heilpädagogischen und kunsttherapeutisch reflektierten Kunstpädagogik Perspektiven und Bedarfe für eine inklusive Weiterentwicklung ableitet. Brenne (2016) griff diese ersten Impulse auf und beschleunigte die Bearbeitung seinerseits aus der Perspektive der künstlerischen Forschung (2017a, 2017b). Die im Jahr 2017 insgesamt festzustellende große Zahl inklusionsbezogener kunstdidaktischer Publikationen ging auf die Tagung „Irgendwie anders“ im Jahr 2016 und die damit verbundene Veröffentlichung des Sammelbandes (Blohm et al. 2017) zurück. Dieser folgt einer Suchbewegung unterschiedlicher Befragungen eines kunstpädagogischen Inklusionsverständnisses, welches mal dem sich aus den poststrukturalistischen, -feministischen und -kolonialen Theorieansätzen begründenden Post-Diskurs folgt, mal der De-Thematisierung von Differenz, indem weniger die Verschiedenheit als vielmehr Gemeinsamkeiten von Kindern in den Fokus gerückt werden und mal dem Gleichheits- und Gerechtigkeitsdiskurs, nach dem marginalisierende und exkludierende schulische Rahmungen in Frage gestellt werden. Hinzu kam der im gleichen Jahr von Sidonie Engels (2017a) veröffentlichte Sammelband „Inklusion und Kunstunterricht“, der sich der Frage der inklusiven Potenziale der künstlerischen Bildung widmete und unter eben dieser Fragestellung zahlreiche Beiträge zum Kunstunterricht versammelt. Sie selbst führt in ihren Beiträgen eine kunstdidaktische Planungsmatrix für eine inklusive Unterrichtsentwicklung ein und leistet damit einen Beitrag zur Überführung kunstpädagogischer Praktiken in eine inklusive Perspektive (Engels 2017b). Im Rahmen dieses Sammelbands gelingt es Bauernschmitt und Sansour (2017), Verbindungslinien zwischen den inklusionsdidaktischen Ansätzen bei Feuser und Seitz und dem Ansatz der künstlerischen Bildung herauszuarbeiten und zugleich die künstlerische Bildung als selbstdifferenzierenden Ansatz mit inklusionsdidaktischem Potenzial herauszustellen, sodass insgesamt hervorzuheben ist, dass sich in Engels 


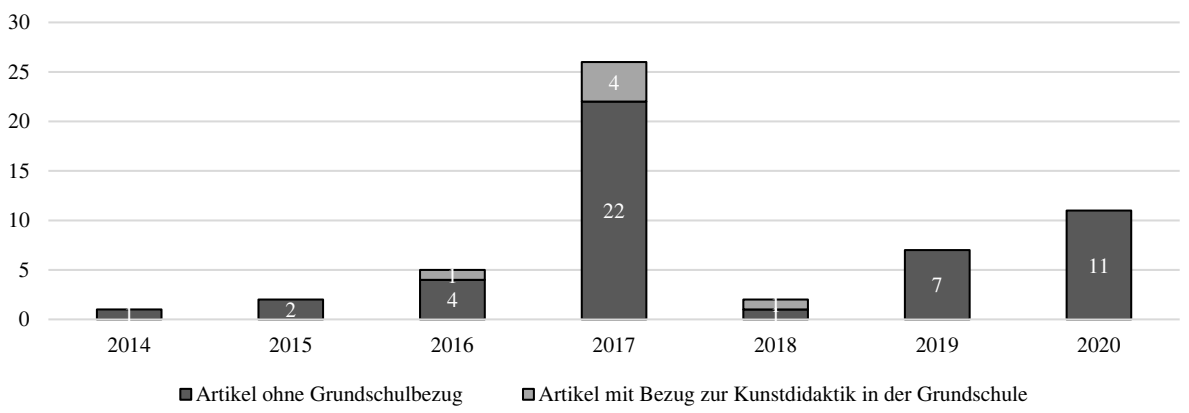

Abb. 1 Beiträge zu einem inklusiven Kunstunterricht

(2017a) Sammelband systematisch inklusiven kunstdidaktischen Fragestellungen genähert wird. Daneben hat Penzel (2017) das Konzept einer ganzheitlichen Lern- und Entwicklungsdiagnostik ausgearbeitet und dabei eine inklusive kunstpädagogische Didaktik und Unterrichtsmethodik in die kunstdidaktische Inklusionsdebatte eingebracht. Dieses angestoßene und sich in den Folgejahren fortsetzende Wachstum der kunstunterrichtlichen Inklusionsforschung führte zu einer, auch nach außen wahrnehmbaren, inklusionspädagogischen Thematisierung der Kunstdidaktik.

So setzte sich der späte Anstoß des kunstunterrichtlichen Inklusionsdiskurses auch im Jahr 2018 mit Einzelbeiträgen fort, die zum einen eine gerechtigkeitstheoretische Fundierung inklusiver Kunstpädagogik anbieten (Sindermann 2018) und zum anderen konkrete Unterrichtssettings thematisieren (Loffredo 2018). An diese schloss sich im Jahr 2019 der Sammelband „In der Praxis“ an (Hornäk et al. 2019), in dessen Fokus Unterrichtsbeispiele zur Umsetzung des Rechts auf inklusive Bildung stehen. Ebenso wurde eine erste Dissertation zu inklusiver Kunstpädagogik vorgelegt (Kaiser 2019). Neben der Weiterentwicklung inklusiver kunstpädagogischer Praktiken durch ein an den Index für Inklusion angelehntes Planungs- und Reflexionsinstrument wird ein Schwerpunkt auf die inklusionsbezogene Professionalisierung von angehenden Kunstlehrkräften gelegt (ebd.). Die hier angestoßene Forschung zur inklusionsbezogenen Professionalität von Kunstlehrkräften wurde von Kaiser und Brenne (2020) in ein kunstpädagogisches Professionalisierungsmodell überführt. Der Kreis der publizierenden Autorinnen und Autoren bleibt jedoch bis in das Jahr 2020 überschaubar, was auch die Beiträge des in diesem Jahr erscheinenden Sammelbands „Die Bildung Aller - Kunstpädagogik und Inklusion“ (Brenne und Kaiser 2020) offenlegen, die sich im Spannungsfeld von erziehungswissenschaftlicher, kunstdidaktischer und fachlicher Kontextualisierung bewegen. Primär setzen sie einen Schwerpunkt auf die Explikation inklusiver kunstpädagogischer Unterrichtsmodelle und so scheint es, dass Inklusion in der Breite des Kunstunterrichts zunehmend angekommen ist. Trotz solcher Initiativen muss mit einem Blick auf die Rahmenpapiere des Fachverbands für Kunstpädagogik oder auf das Standardwerk „Einführung in die Kunstpädagogik“ (Peez 2018), welches mittlerweile in der fünften Auflage erschienen ist, kritisch bemerkt werden, dass inklusionsbezogene Fragestellungen dort kaum wahrnehmbar vertreten sind. Auch die Fachzeitschrift Kunst+ Unterricht hat es bisher versäumt den Inklusionsdiskurs aus fachlicher Per- 
spektive zu thematisieren. Dennoch ist die Zahl kunstunterrichtlicher Publikationen zum Themenbereich Inklusion seit 2014 kontinuierlich angestiegen, so dass aktuell eine ganze Reihe von deutschsprachigen Publikationen vorliegen, von denen aber nur ein geringer Teil einen Grundschulbezug aufweist (s. Abb. 1).

\subsection{Planungs- und Handlungsmodelle für einen inklusiven Kunstunterricht}

Inzwischen sind auch Planungs- und Reflexionsmodelle für eine inklusive kunstunterrichtliche Praxis entstanden, obwohl sie derzeit - so zeigen es die Unterrichtsbeispiele - noch selten in Bezug auf das fachliche Lernen erprobt und unterrichtsmethodisch diskutiert werden (s. Tab. 1). Entsprechend der Gegenstandsbereiche des Fachs Kunst lässt sich die methodische Planung und Reflexion entlang des Erwerbs gestalterischer und bildanalytischer Kompetenzen beschreiben, die im inklusionspädagogisch rekontextualisierten Kunstunterricht zum einen auf die Heranführung an eine eigenständige Gestaltung durch die kunstanaloge und ästhetisch-forschen-

Tab. 1 Unterrichtsmethodische Settings inklusiver Kunstpädagogik

\begin{tabular}{|c|c|c|c|}
\hline $\begin{array}{l}\text { Gegenstandsbereiche } \\
\text { des Fachs }\end{array}$ & $\begin{array}{l}\text { Unterrichtsmethodisches Arrange- } \\
\text { ment }\end{array}$ & Zielperspektive & Autor*in \\
\hline \multirow[t]{7}{*}{$\begin{array}{l}\text { Gestalterische } \\
\text { Kompetenz }\end{array}$} & Künstlerisches Projekt & \multirow[t]{4}{*}{$\begin{array}{l}\text { Kunstanaloge } \\
\text { Erschließung und } \\
\text { ästhetische } \\
\text { Erfahrbarkeit von } \\
\text { Welt }\end{array}$} & $\begin{array}{l}\text { Bauernschmitt } \\
\text { und Sansour } \\
(2017) ; \text { Engels } \\
(2017 b, 2019)\end{array}$ \\
\hline & Materialbasiertes Arbeiten & & $\begin{array}{l}\text { Hornäk (2019); } \\
\text { Kathke (2017) }\end{array}$ \\
\hline & Atelier- und Werkstattarbeit & & $\begin{array}{l}\text { Kathke (2017); } \\
\text { Penzel (2017) }\end{array}$ \\
\hline & $\begin{array}{l}\text { Einsatz offener Aufgaben mit indivi- } \\
\text { duellen Umsetzungsmöglichkeiten }\end{array}$ & & Penzel (2017) \\
\hline & Künstlerisch-ästhetische Forschung & \multirow{3}{*}{$\begin{array}{l}\text { Ästhetisch- } \\
\text { forschende } \\
\text { Erschließung von } \\
\text { Welt auf Basis } \\
\text { subjektiver Er- } \\
\text { kenntnisinteressen }\end{array}$} & $\begin{array}{l}\text { Brenne }(2017 b) \\
\text { Kaiser }(2020)\end{array}$ \\
\hline & $\begin{array}{l}\text { Kartierende Auseinandersetzung zur } \\
\text { kritischen Auseinandersetzung mit } \\
\text { Kunst }\end{array}$ & & Heil (2017) \\
\hline & Mapping & & Engels (2017b) \\
\hline \multirow[t]{7}{*}{$\begin{array}{l}\text { Bildanalytische } \\
\text { Kompetenz }\end{array}$} & $\begin{array}{l}\text { Unterstütze Kommunikation inner- } \\
\text { halb des künstlerischen Prozesses }\end{array}$ & \multirow{3}{*}{$\begin{array}{l}\text { Erwerb von } \\
\text { differenzversierter } \\
\text { Bildlesekompe- } \\
\text { tenz }\end{array}$} & $\begin{array}{l}\text { Hornäk (2017); } \\
\text { Penzel (2017) }\end{array}$ \\
\hline & Auslegung von Bildern durch Bilder & & Engels (2017a) \\
\hline & $\begin{array}{l}\text { Themenbezogene Bildbetrachtung } \\
\text { anhand von an die Inklusion an- } \\
\text { schlussfähigen Bildmotiven }\end{array}$ & & $\begin{array}{l}\text { Loffredo (2016); } \\
\text { Engels (2017b) }\end{array}$ \\
\hline & $\begin{array}{l}\text { Untersuchung des Einsatzes von } \\
\text { Materialien und Techniken }\end{array}$ & \multirow{4}{*}{$\begin{array}{l}\text { Wechselseitige } \\
\text { Bildrezeption zur } \\
\text { ko-konstruktiven } \\
\text { Erschließung von } \\
\text { Welt }\end{array}$} & Engels (2017b) \\
\hline & $\begin{array}{l}\text { Methoden der Kunstgeschichte und } \\
\text { des Bildumgangs }\end{array}$ & & Engels (2017b) \\
\hline & Kollaborative Bildbetrachtung & & $\begin{array}{l}\text { Fortuna und Uhlig } \\
\text { (2017) }\end{array}$ \\
\hline & $\begin{array}{l}\text { Milieu-, kultur- und geschlechter- } \\
\text { sensible Bildbetrachtung }\end{array}$ & & $\begin{array}{l}\text { Kaiser (2019, } \\
\text { 2020) }\end{array}$ \\
\hline
\end{tabular}


de Erschließung von Welt auf Basis subjektiver Forschungsinteressen zielen und zum anderen auf eine differenzversierte Rezeption von Bildern im weitesten Sinn sowie auf die ko-konstruktive Erschließung von Bildinhalten. Dass ein inklusiver Kunstunterricht sich sowohl im Rahmen des Erwerbs gestalterischer als auch bildanalytischer Kompetenzen realisiert, ist durchaus als ein Plädoyer für Pluralität zu verstehen.

So stellen Engels (2017b) und Bauernschmitt und Sansour (2017) das künstlerische Projekt in den Mittelpunkt ihrer inklusionsdidaktischen Überlegungen zum Kunstunterricht. Von hier ausgehend begründen sie eine werkorientierte und eigenständige Gestaltung, experimentelle Problemlösungen und kritische Reflexionen durch Individualisierung und natürliche Differenzierung in der künstlerischen Erschließung von Welt. In ähnlicher Weise spielt ein selbsttätiger und experimenteller Umgang mit künstlerischen Materialien und Techniken im Rahmen materialbasierten Arbeitens (Hornäk 2019; Kathke 2017) sowie im Rahmen von Atelier- und Werkstattarbeit (Kathke 2017; Penzel 2017) eine Rolle für die didaktische Orientierung am Kind und dessen ästhetische Erfahrungen. Nicht primär die Einübung einer künstlerisch-technischen Expertise, sondern die individuelle Hinwendung zu künstlerischen Themenbereichen, Materialien oder Techniken im Rahmen offener und selbstdifferenzierter Aufgaben wird entsprechend durch den Einsatz von Aufgaben mit individuellen Umsetzungsmöglichkeiten, die an die individuellen Interessen und Lernvoraussetzungen anknüpfen, abgesichert (Penzel 2017).

Sich verstärkt der Person des Kindes zuwendend geht es im Rahmen der künstlerisch-ästhetischen Forschung um die Anerkennung kindlicher Potenziale durch eine ästhetisch-forschende Erschließung von Welt und Umwelt auf der Basis subjektiver Erkenntnisinteressen (Brenne 2016, 2017a; Kaiser 2019, 2020). Das selbstbestimmte Lernen der Kinder steht im Vordergrund und geht von ihrer aktiven Partizipation in der Realisierung des Kunstunterrichts aus, auch und vor allem da die Aneignung von Lebenswelt zentral steht. In ähnlicher Weise zielt auch das Mapping (Engels 2017b) wie die kartierende Auseinandersetzung mit (zeitgenössischer) Kunst (Heil 2017) auf ein kontextualisiertes Verständnis von Wirklichkeit durch die interessengeleitete Sammlung, Verarbeitung und Kategorisierung von Material, das entsprechend lebensweltlicher Bezüge gewählt ist und hierüber individuelle Wege der Aneignung anerkennt. Sowohl die kunstorientierte wie die ästhetisch-forschende Erschließung von Welt auf Basis subjektiver Erkenntnisinteressen zielen auf die Heranführung an eine eigenständige ästhetische Erfahrbarkeit der Wirklichkeit, die in eine selbsttätige Gestaltungspraxis mündet.

Im Hinblick auf den Erwerb bildanalytischer Kompetenzen werden in einem inklusiven Unterricht der Erwerb differenzversierter Bildlesekompetenzen sowie die wechselseitige Bildrezeption zur ko-konstruktiven Erschließung von Welt diskutiert. Denn das entscheidende Potenzial inklusiven kunstunterrichtlichen Lernens ist in diesem Zusammenhang die gemeinsame Auseinandersetzung einer heterogenen Gruppe mit für alle relevanten Fragen- oder Problemstellungen. Die Bandbreite der Zugangsweisen, beispielsweise in der gemeinsamen Untersuchung des Einsatzes von Materialien und Techniken (Engels 2017b), eröffnet in einem inklusionspädagogischen Sinn den Blick für den Reichtum der ,Sache“ (Seitz 2006) und die Erschließung des gemeinsamen Gegenstands (Feuser 1989). Dieser Zugang zur Be- 
trachtung künstlerischer Werke sollte - einem ungleichheitskritischen Verständnis von Inklusion folgend - von einer kultur-, milieu- und geschlechtersensiblen Reflexion hinterlegt sein und diskursiv verhandelt werden (Heil 2017; Kaiser 2019). Die kollaborative Bildbetrachtung schärft so den Blick für individuelle Vielfaltsdimensionen wie Wahrnehmungs-, Darstellungs- und Vorstellungsfähigkeiten und für die Resonanzen zwischen den Vielfaltsdimensionen der Kinder (Fortuna und Uhlig 2017), so dass sich darin zeigt, wie sich die perspektivengebundenen Konstruktionsleistungen der Kinder in der Bilderschließung wechselseitig überschneiden und sich mit der fachlichen Perspektive treffen.

Der differenzversierte Umgang mit Bildern gründet bei Loffredo auf der Bildbetrachtung anhand von an die Inklusion anschlussfähigen Bildmotiven, die auch von Engels (2017b) im Hinblick auf die Rezeption angesprochen werden, wobei Engels (2017b) Diversität in einem ungleichheitskritischen Sinn verhandelt, während Loffredo (2016) dabei explizit auf Differenzkategorien verweist. Auf diese Weise verbindet sich bei Loffredo (2016) ein weiter Inklusionsbegriff mit einer förderschwerpunktspezifischen Rekontextualisierung im Zuge der Bildbetrachtung. Wie die bildnerische Kommunikation unabhängig von der Verbalsprache gestärkt werden kann, beantwortet Engels (2017b) mit der Auslegung von Bildern durch Bilder, woran sich auch Hornäks (2017) und Penzels (2017) Überlegungen zur unterstützenden Kommunikation durch den Einsatz von Piktogrammen anschließen.

\subsection{Inklusiver Kunstunterricht in der Grundschule}

Unter Bezugnahme auf die Konzepte der Multiperspektivität, Multimodalität und Multiproduktivität zeichnen Limper und Loffredo (2016) für den Kunstunterricht in der Grundschule einen transdisziplinären Lernprozess am Beispiel von Insekten nach. Gerade die transdisziplinäre Ausrichtung des Kunstunterrichts sowie die Konzepte der Multiperspektivität, Multimodalität und Multiproduktivität leisten ihrer Einschätzung nach im Projekt einen wichtigen Beitrag, die individuelle Lernausgangslagen zu adressieren. Essig-Dehner et al. (2017) richten ihren Fokus auf die individuellen Konstruktionen (künstlerischer) Wirklichkeit von Kindern einer Grundschulklasse, die sich in ihren Bildsprachen und Lesarten zeigt, und auf die gleichzeitigen Ko-Konstruktionsprozesse in der Erschließung des Gegenstands hier im Projekt „Unterwegs“. Damit schließt das von ihnen vorgestellte künstlerische Projekt für die Grundschule unmittelbar an das von Seitz (2006) postulierte Prinzip von Gemeinsamkeit und Verschiedenheit in der Erschießung des gemeinsamen Gegenstandes an. Das beschriebene Projekt wird auch 2019 von Engels erneut thematisiert. Entlang der Darstellung eines performativen Projekts mit einer dritten Grundschulklasse schildert Simon Christophery (2017), wie die Durchführung von Performances Räume eröffnet, in welche Kinder sich mit ihren unterschiedlichen Lernausgangslagen einbringen können. Auch Fortuna und Uhlig (2017) nehmen Bezug auf den Kunstunterricht in der Grundschule, wenn sie ihre Prämissen eines inklusiven Kunstunterrichts an diesem illustrieren. Sie favorisieren ein Inklusionsverständnis, das die Prämisse für inklusives Lehren und Lernen in der geteilten Bezugnahme auf einen gemeinsamen Gegenstand - unter der Bedingung von Gemeinsamkeiten und Verschiedenheiten - sieht. Die Kunstdidaktik zielt danach da- 
rauf, Resonanzen zwischen den Vielfaltsdimensionen von Kindern zu erkennen und unterrichtlich einzubinden, denn gerade die Mehrperspektivität von Kunst und die Vielfalt der Darstellungs- und Repräsentationsformen bieten dazu zahlreiche Möglichkeiten.

\section{Spannungsfelder und weiterführende Perspektiven}

Kunstdidaktische Inklusionsforschung spielt bisher insgesamt und im Kontext der Grundschule im Besonderen eine marginale Rolle - obschon oder gerade, weil dem Kunstunterricht eine Vorreiterrolle in der inklusionsorientierten Umgestaltung unterrichtlicher Praktiken zugesprochen wird. Doch bezieht man die hier geschilderten kunstdidaktischen Erfahrungen auf die Leitidee der Inklusion, kann man feststellen, dass neben explizit kunstunterrichtlichen Initiativen und Ansätzen bereits empirisch abgesicherte kunstunterrichtliche Methoden Mittel und Wege eröffnen, einen inklusiven Kunstunterricht in der Grundschule zu gestalten. Und obwohl sich neben Mitteln und Wegen genügend (kunst-)pädagogische Gründe hierfür finden, ist nicht zu leugnen, dass inklusives kunstunterrichtliches Lernen in der Grundschule im Hinblick auf Konzeptbildung und Forschung weitestgehend Neuland darstellt und Spannungen hervorruft. Abschließend sollen vier Spannungsfelder herausgestellt werden, die weiterführenden Forschungs- und Handlungsbedarf aufzeigen:

- Im Spannungsfeld von Kunst und Kind - den disziplinären fachlichen und pädagogischen Bezügen des Kunstunterrichts - will ein inklusiver Kunstunterricht einerseits jedem Kind passende Lernangebote für eine günstige Leistungs- und Persönlichkeitsentwicklung unterbreiten, andererseits aber gemeinsames Lernen in Kooperation und Ko-Konstruktion ermöglichen. Grundschullehrkräfte stehen vor der Herausforderung solche vermeintlichen Widersprüchlichkeiten zusammenzuführen. Zukünftige Forschung sollte sich von daher vermehrt der Frage widmen, wie eine hohe Fachlichkeit und die Berücksichtigung von Individualität und Gemeinsamkeit in kunstunterrichtliche Konzepte einfließen können. Entsprechend verlangt ein inklusiver Kunstunterricht Lehrkräften sowohl fachlich wie pädagogisch substanzielle Kompetenzen ab, um die Potenziale des Fachs für einen inklusiven Kunstunterricht voll zur Geltung zu bringen. Und so scheint es an einer Schulform, die basale Lern- und Bildungsprozesse organisiert und in der der Kunstunterricht meist fachfremd unterrichtet wird, umso unerlässlicher, professionalisierte Fachlehrkräfte einzusetzen.

- In der Betrachtung des kunstpädagogischen Diskurses fällt auf, dass förderpädagogisch ausgerichtete Handlungsstrategien über einen langen Zeitraum das Feld der Kunstpädagogik und -didaktik dominiert haben und eine inklusive Debatte verhältnismäßig spät an Fahrt aufgenommen hat. Die gegenwärtige Aufmerksamkeit, die man Fragen der Diversität widmet, adressiert vornehmlich subjekt- und kunstorientierte Methoden, wodurch die Kompetenzorientierung in den Hintergrund gerückt wird. Diese Diskursverschiebungen führen zu einer Modifikation des kunstpädagogischen Bildungsauftrags, da sich kunstpädagogische Bildungsprozesse über eine Kunst- und Subjektorientierung nur erschwerend entlang von 
Bildkompetenzen positionieren lassen. Verschärft wird diese Debatte durch die heil- und sonderpädagogische Berücksichtigung der biografischen Situationen und Bedarfe von Kindern, denen ein Förderbedarf zugewiesen wurde, und einer DeThematisierung von Differenz in vielen inklusiven kunstpädagogischen Konzeptionen. Somit offenbaren sich zwei parallel verlaufende Diskursstränge, die sich ebenso auf handlungspraktischer Ebene widerspiegeln. Angezeigt scheint es folglich, dieses Spannungsfeld von Differenz und Diversität unter Berücksichtigung von Kunst- und Bildorientierung zukunftsbezogen kunstpädagogisch zu bearbeiten. Dabei scheinen rein dekonstruktionsbezogene Angebote der Kunstorientierung bisher noch wenig an die kunstdidaktische Praxis angebunden zu sein. Eine Anschlussfähigkeit könnte sich in einer pädagogischen Diagnostik und differenzierungsorientierten Didaktik erweisen, insofern sich diesbezüglich eine Leerstelle im Rahmen fachdidaktischer und unterrichtsmethodischer Zugänge identifizieren lässt.

- Ein weiteres Spannungsfeld eröffnet sich mit einem Blick auf die Brüche und Brücken zwischen wissenschaftlichen und handlungspraktischen Perspektiven. So finden die bereits konzipierten Planungs- und Reflexionsmodelle für eine inklusive Unterrichtsentwicklung im Fach Kunst bisher kaum Berücksichtigung in kunstdidaktischen Unterrichtsvorschlägen - ihre Rezeption dünnt sich auf dem Feld der grundschulischen Kunstdidaktik abermals aus. Entsprechend sollte es künftig auch darum gehen, vorliegende Ansätze empirisch zu erproben.

- Die Entwicklung einer inklusiven Kunstdidaktik im Allgemeinen und für die Grundschule im Spezifischen beschränkt sich derzeit auf die Initiative weniger publizierender Autorinnen und Autoren, die auf dem Feld der Kunstdidaktik noch wenig Resonanz erfährt. Da es geboten scheint, inklusive unterrichtliche Praktiken in den Blick zu nehmen und das relationale Verhältnis von Lehrperson und Kind als wechselseitig vermittelte kunstdidaktische Praxis zu sehen (Heinzel 2019), bedarf es solcher weiteren forschenden Zugänge.

Funding Open Access funding enabled and organized by Projekt DEAL.

Open Access Dieser Artikel wird unter der Creative Commons Namensnennung 4.0 International Lizenz veröffentlicht, welche die Nutzung, Vervielfältigung, Bearbeitung, Verbreitung und Wiedergabe in jeglichem Medium und Format erlaubt, sofern Sie den/die ursprünglichen Autor(en) und die Quelle ordnungsgemäß nennen, einen Link zur Creative Commons Lizenz beifügen und angeben, ob Änderungen vorgenommen wurden.

Die in diesem Artikel enthaltenen Bilder und sonstiges Drittmaterial unterliegen ebenfalls der genannten Creative Commons Lizenz, sofern sich aus der Abbildungslegende nichts anderes ergibt. Sofern das betreffende Material nicht unter der genannten Creative Commons Lizenz steht und die betreffende Handlung nicht nach gesetzlichen Vorschriften erlaubt ist, ist für die oben aufgeführten Weiterverwendungen des Materials die Einwilligung des jeweiligen Rechteinhabers einzuholen.

Weitere Details zur Lizenz entnehmen Sie bitte der Lizenzinformation auf http://creativecommons.org/ licenses/by/4.0/deed.de. 


\section{Literatur}

Bauernschmitt, S., \& Sansour, T. (2017). Das Potenzial künstlerischer Bildung für inklusiven Unterricht. In S. Engels (Hrsg.), Inklusion und Kunstunterricht. Perspektiven und Ansätze künstlerischer Bildung (S. 29-38). Oberhausen: ATHENA.

BDK (2001). Der Kunstunterricht in der Grundschule. Zwischen kindlicher Persönlichkeitsentwicklung und fachlicher Grundbildung. Ästhetische Probleme und Ziele. Arbeitsgruppe Grundschule im BDK. e. V. BDK-Mitteilungen, 37(2), 2-13.

Bering, K. (2006). Visuelle Kompetenz. Kunst und die Orientierung in kulturellen Kontexten. In J. Kirschenmann, F. Schulz \& H. Sowa (Hrsg.), Kunstpädagogik im Projekt der allgemeinen Bildung (S. 228-238). München: kopaed.

Blohm, M., Brenne, A., \& Hornäk, S. (2017). Irgendwie anders. Inklusionsaspekte in den künstlerischen Fächern und der ästhetischen Bildung. Hannover: fabrico.

Brenne, A. (2004). Ressource Kunst. Künstlerische Feldforschung in der Primarstufe. Münster: Monsenstein \& Vannerdat.

Brenne, A. (2006). Ästhetische Forschung - Revistited. Gedanken über ästhetisch-künstlerische Strategien zur Erforschung von Lebenswelt. In M. Blohm, C. Heil, M. Peters, A. Sabisch \& F. Seydel (Hrsg.), Über ästhetische Forschung. Lektüre zu Texten von Helga Kämpf-Jansen (S. 193-201). München: kopaed.

Brenne, A. (2016). Inklusion und Kunstunterricht. In M. Blohm, A. Brenne \& S. Hornäk (Hrsg.), Kunstpädagogische Stichworte (S. 51-54). Hannover: fabrico.

Brenne, A. (2017a). Inklusion und die künstlerische Feldforschung. In M. Blohm, A. Brenne \& S. Hornäk (Hrsg.), Irgendwie anders. Inklusionsaspekte in den künstlerischen Fächern und der ästhetischen Bildung (S. 195-199). Hannover: fabrico.

Brenne, A. (2017b). Künstlerische Feldforschung. In K. Bering, R. Niehoff \& K. Pauls (Hrsg.), Lexikon der Kunstpädagogik (S. 289-290). Oberhausen: Athena.

Brenne, A., \& Kaiser, M. (2020). Die Bildung Aller. Kunstunterricht und Inklusion. Hannover: fabrico.

Brenne, A., Griebel, C., \& Urlaß, M. (2013). MitEinAnder. Zur Praxis einer partizipatorischen Kunstpädagogik in der Grundschule. München: kopaed.

Buschkühle, C.-P. (2009). Künstlerische Wende in der deutschen Kunstpädagogik. In C.-P. Buschkühle, J. Kettel \& M. Urlaß (Hrsg.), horizonte. Interantionale Kunstpädagogik (S. 207-218). Oberhausen: Athena.

Christophery, S. (2017). Von Kopf bis Fuß bewegt. Ein performatives Projekt in einer inklusiven Grundschulklasse. In S. Engels (Hrsg.), Inklusion und Kunstunterricht. Perspektiven und Ansätze künstlerischer Bildung (S. 31-92). Oberhausen: Athena.

Duncker, L., \& Lieber, G. (2013). Bildliteralität und Ästhetische Alphabetisierung: Konzepte und Beispiele für das Lernen im Vor- und Grundschulalter. München: Kopaed.

Ehmer, H. K. (2003). Zwischen Kunst und Unterricht - Spots einer widersprüchlichen wie hedonistischen Berufsbiografie. Hamburg: Hamburg University Press.

Engels, S. (2015). Kunstdidaktik und Inklusion. BDK-Mitteilungen, 2, 18-20.

Engels, S. (2017a). Inklusion und Kunstunterricht. Perspektiven und Ansätze künstlerischer Bildung. Oberhausen: Athena.

Engels, S. (2017b). Inklusion und Kunstdidaktik heute. In S. Engels (Hrsg.), Inklusion und Kunstunterricht. Perspektiven und Ansätze künstlerischer Bildung (S. 11-28). Oberhausen: Athena.

Engels, S. (2019). Inklusiver Kunstunterricht. Chancen prozess- und produktorientierter Ansätze. In S. Hornäk, S. Henning \& D. Gernand (Hrsg.), In der Praxis: Inklusive Möglichkeiten künstlerischen und kunstpädagogischen Handelns (S. 127-142). München: kopaed.

Essig-Dehner, S., Bauernschmitt, S., Geißler, A., Daumann, S., \& Feser, M. (2017). Unterwegs - im inklusiven Kunstunterricht. In S. Engels (Hrsg.), Inklusion und Kunstunterricht. Perspektiven und Ansätze künstlerischer Bildung (S. 39-52). Oberhausen: Athena.

Feuser, G. (1989). Allgemeine integrative Pädagogik und entwicklungslogische Didaktik. Behindertenpädagogik, 1, 4-48.

Fortuna, F., \& Uhlig, B. (2017). Was wäre, wenn: Inklusiver Kunstunterricht. In J. Krautz (Hrsg.), Beziehungen und Bezogenheiten. Relationalität in Pädagogik, Kunst und Kunstpädagogik (S. 169-189). München: kopaed.

Glas, A., \& Sowa, H. (2006). Gestaltungskompetenz: Begriffsklärung und Beispielfelder. In J. Kirschenmann, F. Schulz \& H. Sowa (Hrsg.), Kunstpädagogik im Projekt der allgemeinen Bildung (S. 249-262). München: kopaed. 
Griebel, C. (2017). Passung sehen, unangepasst leben: Collage als Denkfigur künstlerischer Bildung. In S. Engels (Hrsg.), Inklusion und Kunstunterricht. Perspektiven und Ansätze künstlerischer Bildung (S. 93-108). Oberhausen: Athena.

Heil, C. (2017). Inklusion und aktuelle Kunst. Das Blickregime befremden und Normalität dekonstruieren. In M. Blohm, A. Brenne \& S. Hornäk (Hrsg.), Irgendwie anders. Inklusionsaspekte in den künstlerischen Fächern und der ästhetischen Bildung (S. 209-215). Hannover: fabrico.

Heinzel, F. (2019). Zur Doppelfunktion der Grundschule, dem Kind und der Gesellschaft verpflichtet zu sein - die generationenvermittelnde Grundschule als Konzept. Zeitschrift für Grundschulforschung, 12(2), 275-287.

Hornäk, S. (2017). Gebärde, Symbol und Bild. In M. Blohm \& al (Hrsg.), Irgendwie anders (S. 229-235). Hannover: fabrico.

Hornäk, S. (2019). Meine Kita. In S. Hornäk, S. Henning \& D. Gernand (Hrsg.), In der Praxis. Inklusive Möglichkeiten künstlerischen und kunstpädagogischen Handelns (S. 41-50). München: kopaed.

Hornäk, S., Henning, S., \& Gernand, D. (2019). In der Praxis. Inklusive Möglichkeiten künstlerischen und kunstpädagogischen Handelns. München: kopaed.

Huf, C., \& Schnell, I. (2015). Ungleichheiten und Unverfügbarkeiten - Spannungsfelder inklusiver Bildung im Elementar- und Primarbereich. In C. Huf \& I. Schnell (Hrsg.), Inklusive Bildung in KITA und Grundschule (S. 11-16). Stuttgart: Kohlhammer.

Kaiser, M. (2019). Kunstpädagogik im Spannungsfeld von Inklusion und Exklusion. Oberhausen: Athena.

Kaiser, M. (2020). Gestaltungsfragen inklusiver Kunstpädagogik. Zeitschrift Kunst Medien Bildung. http:// zkmb.de/gestaltungsfragen-einer-inklusiven-kunstpaedagogik/. Zugegriffen: 17. Mai 2020.

Kaiser, M., \& Brenne, A. (2020). Kontingenzbearbeitung - Regulativ inklusiver kunstpädagogischer Professionalisierung. QfI - Qualifizierung für Inklusion, 2(1). https://doi.org/10.21248/QfI.22

Kämpf-Jansen, H. (2001). Ästhetische Forschung. Wege durch Alltag, Kunst und Wissenschaft. Zu einem innovativen Konzept ästhetischer Bildung. Köln: Salon.

Kathke, P. (2017). Material/Materialität. In M. Blohm \& al (Hrsg.), Irgendwie anders (S. 237-241). Hannover: fabrico.

Kirchner, C. (1999). Kinder und Kunst der Gegenwart. Zur Erfahrung mit zeitgenössischer Kunst in der Grundschule. Seelze-Velber: Kallmeyer.

Kirchner, C. (2013). Grundlagen des Kunstunterrichts. In C. Kirchner (Hrsg.), Kunst: Didaktik für die Grundschule (S. 9-35). Berlin: Cornelsen.

Kucharz, D. (2015). Inklusiver Sachunterricht. In C. Huf \& I. Schnell (Hrsg.), Inklusive Bildung in KITA und Grundschule (S. 221-234). Stuttgart: Kohlhammer.

Limper, B., \& Loffredo, A.-M. (2016). Was leistet Ästhetische Bildung in der Grundschule für die Entwicklung der Kognition? - Transdisziplinäres Lernen am Beispiel von Insekten einer 1. Klasse. In A.M. Loffredo (Hrsg.), Kunstunterricht und Inklusion. Eine bildungstheoretische und fachdidaktische Untersuchung gegenwärtiger Anforderungen an ausgewählten Unterrichtsbeispielen für die Primarund Sekundarstufen (S. 227-244). Oberhausen: Athena.

Loffredo, A.-M. (2016). Kunstunterricht und Inklusion. Eine Annäherung. In A.-M. Loffredo (Hrsg.), Kunstunterricht und Inklusion. Eine bildungstheoretische und fachdidaktische Untersuchung gegenwärtiger Anforderungen an ausgewählten Unterrichtsbeispielen für die Primar- und Sekundarstufen (S. 11-57). Oberhausen: Athena.

Loffredo, A.M. (2018). Inklusion aus fachdidaktischer Perspektive begreifen. BDK-Mitteilungen, 4, 26-31.

Lütje-Klose, B. (2018). Überlegungen zur Konzeptualisierung von Inklusion - Einordnungen, Ansätze, Hinweise zur Operationalisierung. In M. Walm, T. Häcker, F. Radisch \& A. Krüger (Hrsg.), Empirisch-pädagogische Forschung in inklusiven Zeiten. Konzeptualisierung, Professionalisierung, Systementwicklung (S. 27-50). Bad Heilbrunn: Klinkhardt.

Peez, G. (2018). Einfuihrung in die Kunstpädagogik. Stuttgart: Kohlhammer.

Penzel, J. (2017). Inklusion und Kunstunterricht. http://www.integrale-kunstpaedagogik.de/assets/ikp_ um_inklusion_2018.pdf. Zugegriffen: 12. Aug. 2020.

Seitz, S. (2006). Inklusive Didaktik: Die Frage nach dem ,Kern der Sache‘. http://www.inklusion-online. net/index.php?menuid=3\&reporeid=16. Zugegriffen: 23. Juni 2017.

Seitz, S. (2008). Leitlinien didaktischen Handelns. Zeitschrift für Heilpädagogik, 59(6), 226-233.

Seitz, S. (2020). Dimensionen inklusiver Didaktik - Personalität, Sozialität und Komplexität. Zeitschrift für Inklusion, (2). https://www.inklusion-online.net/index.php/inklusion-online/article/view/570. Zugegriffen: 17. Febr. 2021. 
Sindermann, M. (2018). Inklusive Kunstpädagogik - potenzial- und differenzaffin. Zeitschrift für Inklusion (1). https://www.inklusion-online.net/index.php/inklusion-online/article/view/428. Zugegriffen: 30. Apr. 2018.

Sowa, H. (2017). Differente Herstellungsverfahren verkörperter Raumimagination in den bildenden Künsten. Zur enaktivistischen Grundlegung und gestaltungsbezogenen Ausdifferenzierung der dreidimensionalen Gestaltungsdidaktik in der Kunstpädagogik. In H. Sowa, M. Miller \& S. Fröhlich (Hrsg.), Bildung der Imagination (Bd. 3, S. 315-348). Oberhausen: Athena.

Staudte, A. (1993). Ästhetische Erziehung und Kunst. Lernen zwischen Sinnlichkeit, Kreativität und Vernunft. In D. Haarmann (Hrsg.), Handbuch Grundschule (S. 292-303). Weinheim: Beltz.

Theunissen, G. (2013). Kunst als Ressource in der Behindertenarbeit. Marburg: Lebenshilfe.

Uhlig, B. (2005). Kunstrezeption in der Grundschule. Zu einer grundschulspezifischen Rezeptionsmethodik. München: kopaed.

Urlaß, M., Brenne, A., \& Griebel, C. (2012). Partizipatorische Kunstpädagogik in der Grundschule. Einführung. In A. Brenne, A. Sabisch \& A. Schnurr (Hrsg.), revisit (S. 93-96). München: kopaed.

Vogt, M. (2019). Grundlegende Bildung als Zielvorgabe einer Schule für alle - Deutungsvarianten in der Geschichte der Grundschule in Deutschland. Zeitschrift für Grundschulforschung, 12(2), 241-258.

Winderlich, K. (2019). lernen. grund_schule kunst bildung, Bd. 7. Oberhausen: Athena.

Wocken, H. (2015). Das Haus der inklusiven Schule. Baustellen - Baupläne - Bausteine. Hamburg: Feldhaus.

Ziemen, K. (2014). Inklusion und deren Herausforderungen für die (Fach-)Didaktik. In B. Arnheim, B. \& M. Dziak-Mahler (Hrsg.), Fachdidaktik inklusiv (S. 45-56). Münster: Waxmann. 